\section{Prognostic Impact of Inferior Mesenteric Artery Lymph Node Metastasis in Colorectal Cancer}

\section{TO THE EDITORS:}

We read with interest the article of Kang et al. concerning the prognostic significance of inferior mesenteric artery lymph node metastases in rectal and sigmoid cancer patients. ${ }^{1}$ We are pleased that the anatomic distribution of lymph node metastasis in colorectal cancer is gaining importance, and this article also suggests that it may also influence 5-year survival. The authors divided patients with rectal or sigmoid cancer in two groups (with or without inferior mesenteric lymph node involvement) and concluded that patients with inferior mesenteric lymph node metastases had a higher percentage of systemic metastases and far worse prognosis compared with the group with no metastases.

We want to express some thoughts about the issues discussed. Data for local recurrence rate show that both isolated $(0.0 \%$ vs. $4.4 \%)$ and overall local recurrence rate $(3.0 \%$ vs. $8.6 \%)$ was worse in the group with no inferior mesenteric lymph node involvement. Because involved lymph nodes may act not only as predictors of systemic failure but also as foci for local recurrence, these results are difficult to explain. It would be easier to accept higher local recurrence rates in the group with involved lymph nodes. Taking into consideration that the overall lymph node collection yield was similar but that there was a marked difference in the number removed from the inferior mesenteric root between the two groups, one may hypothesize that the presence of enlarged lymph nodes in the area of inferior mesenteric artery in patients with nodal metastases had influenced the surgical technique and led to a more meticulous dissection of this area. Otherwise, the higher local recurrence rate in the group of patients with no metastases to the mesenteric root remains unaccounted for.

The authors stated that inferior mesenteric root involvement was a prognostic factor for para-aortic and systemic metastases. Data that could reinforce this argument could come from the study of stage IV rectal and sigmoid patients of their database. If we accept the hypothesis that inferior mesenteric lymph nodes are a getaway for systemic metastases, then it is expected that patients with stage IV disease at the time of diagnosis who had been operated on with curative intent (operable liver or peritoneal metastases) will have a far higher percentage of involved inferior mesenteric lymph nodes. These data are missing from the presented data. In this context, rectal cancer patients who have been subjected to preoperative radiochemotherapy and who have advanced-stage disease also may have a higher percentage of involved inferior mesenteric lymph nodes. These data are also missing from this article.

The authors concluded that, on the basis of the findings of their study, inferior mesenteric nodal metastasis was associated with high incidence of systemic recurrence and poor prognosis. In our opinion, this notion, in conjuction with articles that suggest no survival benefit from the high ligation of inferior mesenteric artery, could imply that inferior mesenteric artery nodal metastases must be classified under $\mathrm{M}$ and not under $\mathrm{N}$ stage in the tumor, node, metastasis system of classification as a result of their strong prognostic but poor therapeutic impact. This assumption remains open for debate if we accept the results of this article.

Panagiotis Taflampas, MD ${ }^{\mathbf{1}}$, Manousos Christodoulakis, $\mathrm{MD}^{2}$, and Eelco DeBree ${ }^{2}$

${ }^{1}$ B' Surgical Department, Venizeleio Hospital, Herakleion, Crete, Greece;

${ }^{2}$ Department of Surgical Oncology, University Hospital of Crete, Herakleion, Crete, Greece

e-mail: taflampas2003@yahoo.gr

Published Online: 7 January 2011

(C) Society of Surgical Oncology 2011

\section{REFERENCE}

1. Kang J, Hur H, Soh B, et al. Prognostic impact of inferior mesenteric artery lymph node metastasis in colorectal cancer. Ann Surg Oncol. 2010 (in press). 